\title{
As Relações entre o Uso de Jogos Eletrônicos, Personalidade e o Bem-Estar de Jogadores
}

\author{
Fábio Spricigo Coser $^{1}$, Claudia Hofheinz Giacomoni \\ Universidade Federal do Rio Grande do Sul, Porto Alegre-RS, Brasil
}

\section{RESUMO}

Jogos eletrônicos são populares em várias partes do mundo. Pesquisas sobre os efeitos deles sobre a saúde dos jogadores vêm sendo realizadas desde a criação dos videogames. Este estudo investigou as relações entre a frequência de jogo, os fatores de personalidade, os gêneros de jogos e as variáveis de bem-estar subjetivo. A pesquisa foi respondida por 759 participantes. Foram encontradas diferenças significativas nas variáveis Satisfação de Vida, Afetos Positivos, Extroversão e Realização entre jogadores e não jogadores. As correlações entre Horas de Jogo e Satisfação de Vida, Afetos Positivos e Realização foram baixas. Horas de Jogo por Semana não é um forte preditor do Bem-Estar Subjetivo. Motivações, paixão e necessidades psicológicas básicas do jogador podem ter maior impacto nas variáveis de bem-estar. Os resultados apontados neste estudo vão contra a concepção de que o tempo de jogo, individualmente, seja responsável por alterações nos níveis de bem-estar.

Palavras-chave: psicologia positiva; videogame; bem-estar subjetivo; jogos digitais.

\section{ABSTRACT - The Relations between Video Games, Personality and Players' Well-Being}

Video games are popular in most parts of the world. Studies about their effects on the players' health have been produced since their creation. This study investigated the relations between subjective well-being and game frequency, personality traits and game genres. The questionnaire was completed by 759 participants. Significant differences were found between players and non-players in relation to Life Satisfaction, Positive Affect, Extraversion and Conscientiousness. Correlations between Gaming Hours per Week, Positive Affect, Life Satisfaction and Conscientiousness were weak. Correlations between Game Genre and Personality Traits were weak. Gaming Hours per Week was not a strong predictor of Subjective Well-Being. Other variables should be considered when researching gamers' well-being. Motivations, passion and basic psychological needs may have a higher impact on well-being variables. The results found in this study go against the idea that the frequency of gaming, individually, is responsible for alterations in well-being scores.

Keywords: positive psychology; video game; subjective wellbeing; gamers.

\section{RESUMEN - Las Relaciones entre los Videojuegos, Personalidad y el Bienestar de Jugadores}

Los videojuegos son populares en varias partes del mundo. Las investigaciones sobre los efectos de los mismos sobre la salud de los jugadores vienen siendo realizadas desde la creación de los juegos electrónicos. Esta investigación evaluó las relaciones entre la frecuencia de juego, los factores de personalidad, los géneros de juegos y las variables de bienestar subjetivo. La encuesta fue realizada con 759 participantes. Se encontraron diferencias significativas en las variables Satisfacción con la Vida, Afectos Positivos, Extroversión y Realización entre jugadores y no jugadores. Las correlaciones entre Horas de Juego y Satisfacción con la Vida, Afectos Positivos y Realización fueron bajas. Horas de Juego por Semana no es un fuerte predictor del Bienestar Subjetivo. Los resultados señalados en este estudio van en contra de la concepción de que el tiempo de juego, individualmente, es responsable de alteraciones en los niveles de bienestar.

Palabras clave: psicología positiva; videojuegos; bienestar subjetivo; jugadores.

Encontrar a felicidade e viver de forma plena é uma preocupação humana desde os tempos antigos. Filósofos gregos abordaram o conceito de "boa vida" por meio da eudaimonia e hedonia. A primeira faz referência a uma existência com propósito e significado, vivendo de forma virtuosa e significativa para a comunidade. Já a segunda refere-se à busca por sensações prazerosas e atividades que tragam emoções positivas ao indivíduo (Delle Fave, Massimini, \& Bassi, 2011). Alguns autores buscaram definir um construto referente ao julgamento de uma pessoa quanto a sua própria existência, levando em consideração os aspectos emocionais e de satisfação com sua vida. Esse construto foi denominado Bem-Estar Subjetivo (BES) (Diener, 1984; Diener et al., 2017).

O BES se aproxima da linha hedonista de pensamento sobre a felicidade, focando na experiência subjetiva do indivíduo. O construto é composto por dois componentes, cognitivo e afetivo (Diener, 1984). O primeiro é referente ao quão satisfeito um sujeito está com sua vida, enquanto o segundo engloba os sentimentos 
positivos e negativos que uma pessoa vivencia no seu cotidiano (Diener et al., 2017). O bem-estar é amplamente estudado, assim como sua relação com elementos sociais, econômicos e psicológicos (Diener, Tay, \& Oishi, 2013; Błachnio, Przepiorka, \& Pantic, 2016; Diener et al., 2017). A qualidade das relações sociais, autoestima, satisfação no trabalho e personalidade são fatores que se relacionam com os níveis de bem-estar (Błachnio et al., 2016; Diener et al., 2017).

A personalidade está relacionada com o modo como um indivíduo sente, age e interage com o mundo em sua volta. A teoria dos Cinco Grandes Fatores ou Big Five propõe que a personalidade de cada pessoa é composta por cinco facetas principais que se mantêm relativamente estáveis ao longo da vida (McCrae \& Costa, 2008; Anglim \& Grant, 2016). Extroversão, o quanto uma pessoa se beneficia do contato com outras pessoas; Socialização ou Amabilidade, que se refere a quanto um indivíduo confia, é tolerante e investe em relações mais profundas com outros; Realização ou Conscienciosidade representa a capacidade de organização, praticidade e perseverança; Neuroticismo, um indicador de saúde mental, propensão a experenciar emoções negativas; e Abertura à Experiência, que rege a capacidade criativa e de assimilar mudanças (McCrae \& Costa, 2008). Alguns desses fatores de personalidade possuem fortes relações com o bem-estar (Soto, 2015; Anglim \& Grant, 2016). Neuroticismo é o fator que correlaciona mais fortemente com satisfação de vida e afetos negativos, enquanto extroversão relaciona-se com os afetos positivos (Anglim \& Grant, 2016). Alguns pesquisadores investigam as relações do bem-estar e fatores de personalidade com o engajamento a certas atividades, como o uso de jogos eletrônicos (Lehenbauer-Baum et al., 2015; Braun, Stopfer, Müller, Beutel, \& Egloff, 2016; Jimenez et al., 2019).

O uso de jogos eletrônicos é um dos meios de entretenimento mais populares do mundo. Pessoas de todas as faixas etárias os utilizam para relaxar, distrair-se dos problemas da vida e entrar em contato com outros jogadores (Vella, Johnson, \& Hides, 2015; Comello, Francis, Marshall, \& Puglia, 2016; Allen \& Anderson, 2018). As discussões sobre jogos eletrônicos e seus impactos em questões psicológicas vêm se mostrando presentes na literatura (Braun et al., 2016; Kaye, Carlisle \& Griffiths, 2017; Lobel, Engels, Stone, Burk, \& Granic, 2017; Jimenez et al., 2019). Apesar de grande parte dos estudos focarem em aspectos negativos como sintomas de adição e uso problemático, alguns autores pesquisam os potenciais dos videogames para a saúde física e mental (Granic, Lobel, \& Engels, 2013; Lobel et al., 2017). Quando utilizados como intervenções com propósitos específicos (Serious Games), os jogos se mostraram naturalmente engajadores, potencializando seus efeitos benéficos (Granic et al., 2013). Porém, é importante investigar seu uso como uma atividade de lazer, devido a sua popularidade entre pessoas de todas as idades e gêneros.
Estudos que investigaram relações diretas entre bem-estar e o tempo de engajamento aos videogames reportaram resultados variados. Pesquisadores apontaram correlações positivas, negativas ou inexistentes entre as variáveis de bem-estar e as horas de jogo (Comello et al., 2016; Carras et al., 2017; Allen \& Anderson, 2018). A personalidade dos jogadores também foi alvo de investigações (Lehenbauer-Baum et al., 2015; Braun et al., 2016; Jimenez et al., 2019). Em pessoas que reportaram sintomas de transtorno do jogo pela internet, neuroticismo se mostrou mais alto, enquanto realização, abertura, extroversão e amabilidade obtiveram escores mais baixos (Lehenbauer-Baum et al., 2015; Braun et al., 2016). Relações também foram encontradas entre os fatores de personalidade e os gêneros de jogos (Braun et al., 2016; Jimenez et al., 2019). Maiores níveis de extroversão e menores de neuroticismo foram associados com o uso de jogos de ação e multijogadores (multiplayer) (Braun et al., 2016; Jimenez et al., 2019). Enquanto a preferência por Role-Playing Games (RPGs) correlacionou-se positivamente com maiores escores de neuroticismo (Braun et al., 2016).

Não há consenso entre os pesquisadores sobre a influência da personalidade na escolha dos jogos eletrônicos, e como eles influenciam as variáveis de bem-estar. $\mathrm{O}$ objetivo deste estudo foi verificar a existência de relações entre essas variáveis na população brasileira. Investigouse as correlações entre os traços de personalidade e os gêneros de jogos eletrônicos, comparou-se os níveis de bem-estar de jogadores e não jogadores e explorou-se a relação entre o bem-estar subjetivo e os fatores de personalidade com o tempo dedicado semanalmente aos jogos eletrônicos. Consideram-se as seguintes hipóteses: A personalidade dos jogadores possui relação com as preferências por gêneros de jogos eletrônicos (Braun et al., 2016; Jimenez et al., 2019). Os escores de afetos positivos e satisfação de vida são maiores em jogadores que se dedicam aos jogos eletrônicos, em comparação com os que não jogam (Allen \& Anderson, 2018). A frequência de jogo, em horas semanais, relaciona-se com as variáveis de bem-estar subjetivo e com fatores de personalidade (Braun et al., 2016; Allen \& Anderson, 2018). Esse estudo investigou os fatores de personalidade, escores de bem-estar subjetivo, preferências por gênero de jogo eletrônico e dados demográficos dos participantes.

\section{Método}

\section{Participantes}

A amostra foi composta por 599 participantes que utilizam jogos eletrônicos por pelo menos uma hora por semana e 160 que não possuem o hábito de jogar. A amostra final foi de 759 indivíduos com idades entre 18 e 70 anos $(M=27,12 ; D P=6,76)$. Todos os participantes são brasileiros, sendo a maior parte $(69,9 \%)$ da região Sul do Brasil (59,4\% do estado Rio Grande do Sul), seguido 
pelas regiões Sudeste (17,8\%), Nordeste (7,3\%), CentroOeste $(3,9 \%)$ e Norte $(1,2 \%)$. Os participantes que jogavam menos de uma hora por semana foram considerados como não jogadores e possuem idade média de 29,51 anos, são $87,5 \%$ do gênero feminino. Os indivíduos que dedicam mais de uma hora por semana ao uso de videogames foram considerados jogadores. A idade média foi de 26,8 anos, $76,5 \%$ do gênero masculino. Posteriormente, os participantes foram separados em dez grupos com base nos desvios padrões das horas de jogo. O número de grupos foi escolhido de modo a evitar que jogadores que apresentassem diferenças de mais de 10 horas de jogo por semana fossem categorizados nos mesmos grupos, visto que a experiência de jogo poderia ser divergente entre jogadores. Portanto, optou-se por não prejudicar as análises dos jogadores que dedicam até 25 horas semanais de jogo, os quais são a maior parte da amostra, em detrimento das comparações com participantes que dedicam de 25 até 74 horas, devido à baixa amostra de jogadores com esse perfil.

\section{Instrumentos}

Para realização desta pesquisa, foram utilizados os seguintes instrumentos: questionário sociodemográfico, questionário de Perfil de Jogador, Escala Reduzida de Descritores de Personalidade (Natividade \& Hutz, 2015), Escala de Satisfação de Vida (Hutz, Zanon, \& Bardagi, 2014) e a Escala de Afetos Positivos e Negativos (Zanon, Bastianello, Pacico, \& Hutz, 2013).

O questionário sociodemográfico foi composto por questões que investigaram o perfil social do participante. Foram realizadas perguntas sobre gênero, idade, escolaridade e localidade. O Questionário de Perfil de Jogador foi desenvolvido pelos pesquisadores. Buscou-se investigar o tempo dos participantes de dedicação a jogos eletrônicos, plataforma (console, smartphone/tablet, computador) e interação com outros jogadores (preferências por jogos multiplayer ou single player). Com base nos dez focos da faceta Gameplay propostos por Lee et al. (2014) e em outros tipos propostos pela literatura (Zammitto, 2010; Scharkow, Festl, Vogelgesang, \& Quandt, 2015), o questionário abordou a preferência de jogadores aos seguintes gêneros de jogos: Ação, Aventura, Corrida, Luta, Puzzle, Role-Playing Games (RPG), Tiro, Simulação, Esporte, Estratégia, Música e Ritmo (M\&R), Sandbox, On-line - Multiplayer On-line Battle Arena (MOBA) e On-line - Massively Multiplayer Online Role-Playing Game (MMORPG).

A preferência foi avaliada de um "não gosto" a cinco "gosto muito", uma opção "não conheço esse gênero" também foi adicionada para cada item. Ao lado de cada tipo de jogo foram disponibilizados exemplos de jogos que pertencem ao gênero par melhor compreensão. Os participantes também foram requisitados a escolher um dos 14 gêneros da lista como seu favorito.

A Escala Reduzida de Descritores da Personalidade (Red-5) de Natividade e Hutz (2015) busca avaliar os participantes por meio do modelo dos Cinco Grandes Fatores da Personalidade, também conhecido como Big Five (McCrae \& Costa, 2008). Essa escala é composta por 20 itens em forma de adjetivos ou pequenas expressões e pontua as respostas nos fatores extroversão $(\alpha=0,84)$, socialização $(\alpha=0,77)$, abertura à experiência $(\alpha=0,59)$, realização $(\alpha=0,70)$ e neuroticismo $(\alpha=0,67)$. O participante responde o quão bem cada item o descreve por meio de uma escala de sete pontos. Os coeficientes de consistência interna e correlação teste-reteste sugerem que a escala é adequada para uso (Natividade $\&$ Hutz, 2015).

O bem-estar subjetivo foi avaliado utilizando-se as escalas Escala de Satisfação de Vida (Hutz et al., 2014) e a Escala de Afetos Positivos e Negativos (Zanon et al., 2013). A primeira é a versão adaptada para o Brasil da Satisfaction With Life Scale (Diener, Emmons, Larsen, \& Griffin, 1985). Trata-se de uma escala de cinco itens que visa medir a satisfação do participante com a sua vida atual. Para responder, deve-se marcar um valor que varia de um (concordo plenamente) a sete (discordo plenamente) para cada uma das afirmações. A escala original apresenta consistência interna adequada, possuindo um valor alpha de 0,87 .

A Escala de Afetos Positivos e Negativos foi publicada no Brasil por Zanon et al. (2013). Trata-se de uma escala com dois fatores, afetos positivos e afetos negativos. O instrumento é composto por 20 itens no formato de adjetivos, com dez itens para cada fator. O respondente deve indicar o quanto tem sentido cada um dos afetos em uma escala de um (nem um pouco) a cinco (extremamente). Os índices de consistência interna dessa escala são de 0,88 para afetos positivos e 0,86 para afetos negativos, sendo considerados adequados para utilização, mantendo as propriedades psicométricas da escala original.

\section{Procedimentos}

A amostra foi não probabilística, e os participantes foram recrutados utilizando-se de um critério de conveniência, por meio de redes sociais, fóruns e comunidades de jogos eletrônicos. Os instrumentos foram respondidos de forma on-line, por meio da plataforma SurveyMonkey. 924 participantes responderam ao formulário. Respostas incompletas, respondentes com menos de 18 anos de idade e outliers foram removidos. Aos respondentes, foi apresentado um Termo de Consentimento Livre e Esclarecido informando sobre a proposta do estudo e os riscos envolvidos. Essa pesquisa foi autorizada pelo Comitê de Ética em Pesquisa do Instituto de Psicologia da Universidade Federal do Rio Grande do Sul e se encontra registrado sob o CAAE No 86085518.8.0000.5334.

\section{Análise de dados}

Foram realizadas estatísticas descritivas para investigar o perfil da amostra. Os participantes foram divididos em 10 grupos, com os quais foram realizadas as análises de variância (ANOVA) para investigar as diferenças nos 
escores de bem-estar subjetivo e fatores de personalidade. Procedimentos post-hoc foram realizados utilizando o teste de Bonferroni. Adicionalmente, foram realizadas correlações de Pearson para identificar se os fatores da personalidade relacionam-se com a preferência por gênero de jogos eletrônicos.

\section{Resultados}

Jogadores responderam questões sobre seus hábitos e preferências relacionadas a jogos eletrônicos, como: plataformas utilizadas para jogar, preferência por gêneros de jogos e engajamento em jogos singleplayer (jogador único), multiplayer on-line (multiplos jogadores por meio da rede) ou multiplayer local (múltiplos jogadores no mesmo ambiente físico). Os participantes dedicam de uma a 74 horas por semana aos jogos eletrônicos $(M=14,58, D P=13,09)$ e possuem em média 2,25 plataformas nas quais jogam videogames. $\mathrm{O}$ equipamento considerado mais popular entre os participantes foi o computador $(71 \%)$, seguido pelos smartphones (51\%) (Figura 1).

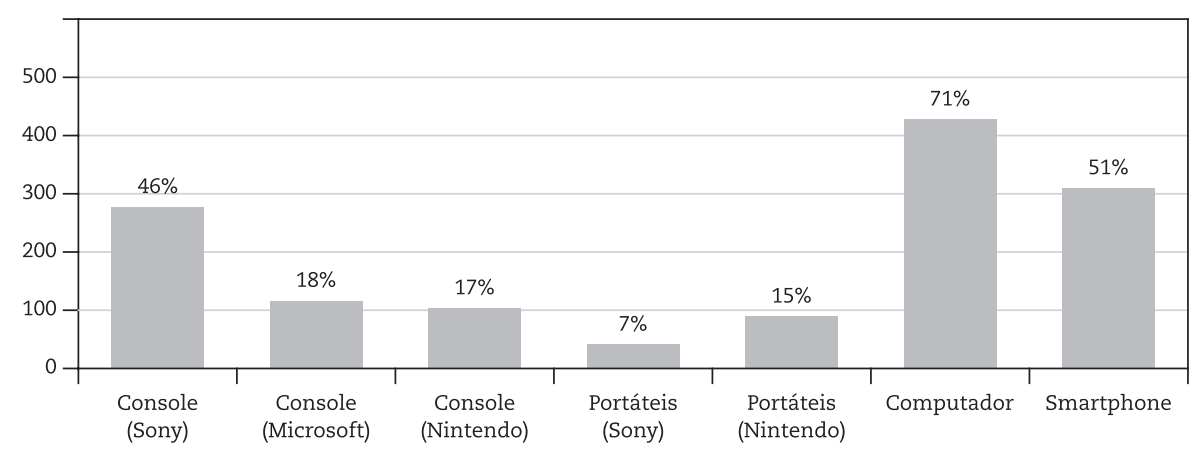

Figura 1. Frequência de plataformas de jogo

Não houve correlações entre o número de plataformas e as horas de jogo semanais (HJs). Porém, HJs relacionou-se positivamente com a preferência por alguns gêneros de jogo: RPGs $(r=0,188, p<0,01)$, Estratégia $(r=0,117, p<0,01)$, Sandbox $(r=0,117, p<0,05)$, MOBAs $(r=0,186, p<0,01)$ e MMORPGs $(r=0,304, p<0,01)$. Quanto aos gêneros favoritos, RPG foi o mais votado por $24,9 \%$ dos participantes, seguido por Aventura (15,7\%) e MMORPG (13,9\%) (Figura 2).

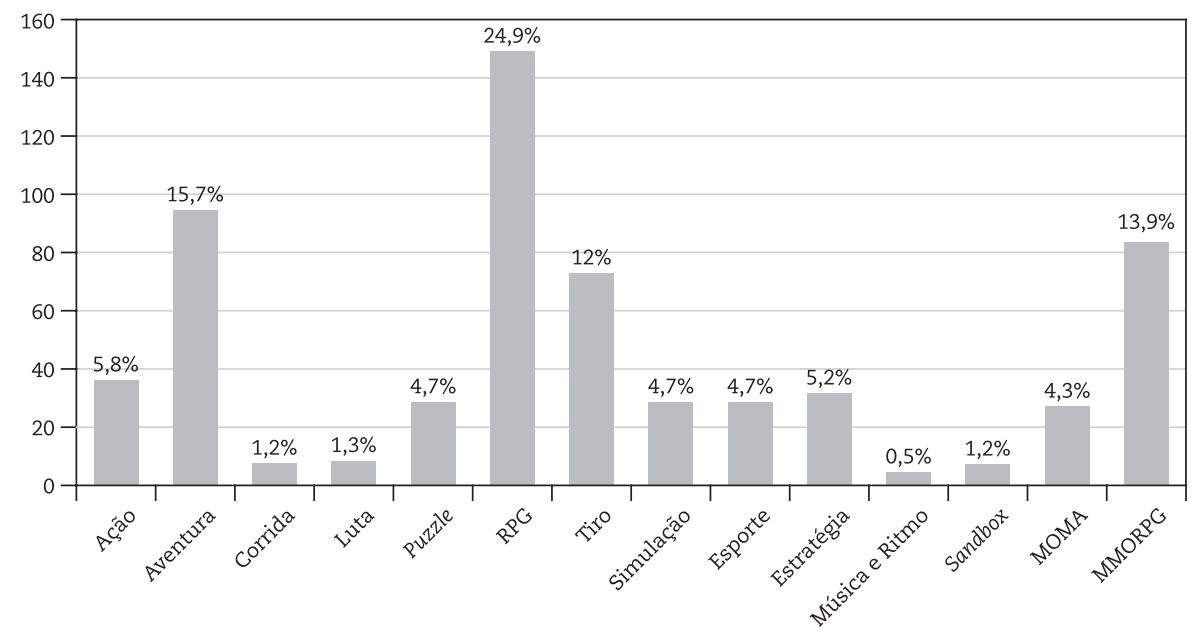

Figura 2. Preferência por gênero de jogo 
Algumas correlações entre a preferência por gêneros foram significativas, como o uso de MMORPGs que apresentou relações com MOBAs, RPGs e Estratégia; jogos de Ação com Aventura, Corrida e Luta; RPGs com Aventura, Estratégia e relação negativa com Corrida e Esporte; entre outros (Tabela 1). Quando correlacionados com os fatores de personalidade, como apresentado na Tabela 2, Abertura à Experiência foi o fator que apresentou relação significativa com o maior número de gêneros de jogo (Positiva: Aventura, Puzzle, Estratégia, Música e Ritmo; Negativa: Esporte). Foi seguida por Neuroticismo e Socialização (com quatro relações cada) e por Extroversão e Realização (com três relações cada).

Tabela 1

Correlações das Preferências entre Diferentes Gêneros de Jogo

\begin{tabular}{|c|c|c|c|c|c|c|c|}
\hline Gêneros & 1 & 2 & 3 & 4 & 5 & 6 & 7 \\
\hline 1. Ação & 1 & & & & & & \\
\hline 2. Aventura & $0,351^{* *}$ & 1 & & & & & \\
\hline 3. Corrida & $0,327^{* *}$ & $0,120^{* *}$ & 1 & & & & \\
\hline 4. Luta & $0,384^{* *}$ & $0,210^{* *}$ & $0,345^{* *}$ & 1 & & & \\
\hline 5. Puzzle & $0,177^{* *}$ & 0,009 & $0,112^{* *}$ & 0,051 & 1 & & \\
\hline 6. RPG & 0,060 & $0,347^{* *}$ & $-0,110^{* *}$ & 0,033 & $-0,013$ & 1 & \\
\hline 7. Tiro & $-0,016$ & $0,113^{* *}$ & $0,147^{* *}$ & $0,149^{* *}$ & $-0,138^{* *}$ & $-0,016$ & 1 \\
\hline 8. Simulação & $-0,002$ & $-0,044$ & $0,099^{*}$ & $-0,051$ & $0,172^{* *}$ & 0,069 & $-0,069$ \\
\hline 9. Esporte & 0,061 & 0,033 & $0,318^{* *}$ & $0,148^{* *}$ & $-0,078$ & $-0,220^{* *}$ & $0,194^{* *}$ \\
\hline 10. Estratégia & $-0,034$ & 0,067 & $-0,014$ & $-0,021$ & 0,068 & $0,286^{* *}$ & ,098* \\
\hline 11. $M \& R$ & $0,173^{* *}$ & $0,105^{*}$ & $0,191^{* *}$ & $0,160^{* *}$ & $0,217^{* *}$ & 0,052 & $-0,007$ \\
\hline 12. Sandbox & 0,022 & 0,079 & $-0,025$ & $-0,106^{*}$ & $0,107^{*}$ & $0,202^{* *}$ & $0,115^{* *}$ \\
\hline 13. MOBA & $-0,071$ & $0,114^{* *}$ & $-0,104^{*}$ & 0,006 & $-0,047$ & $0,188^{* *}$ & $0,132^{* *}$ \\
\hline 14. MMORPG & $-0,028$ & $0,128^{* *}$ & $-0,081$ & 0,014 & $-0,050$ & $0,505^{* *}$ & $0,092^{*}$ \\
\hline Gêneros & 8 & 9 & 10 & 11 & 12 & 13 & 14 \\
\hline \multicolumn{8}{|l|}{ 1. Ação } \\
\hline \multicolumn{8}{|l|}{ 2. Aventura } \\
\hline \multicolumn{8}{|l|}{ 3. Corrida } \\
\hline \multicolumn{8}{|l|}{ 4. Luta } \\
\hline \multicolumn{8}{|l|}{ 5. Puzzle } \\
\hline \multicolumn{8}{|l|}{ 6. RPG } \\
\hline \multicolumn{8}{|l|}{ 7. Tiro } \\
\hline 8. Simulação & 1 & & & & & & \\
\hline 9. Esporte & 0,057 & 1 & & & & & \\
\hline 10. Estratégia & $0,322^{* *}$ & $0,082^{*}$ & 1 & & & & \\
\hline 11. $M \& R$ & $0,224^{* *}$ & 0,054 & $0,083^{*}$ & 1 & & & \\
\hline 12. Sandbox & $0,288^{* *}$ & $-0,072$ & $0,228^{* *}$ & $0,148^{* *}$ & 1 & & \\
\hline 13. MOBA & $0,085^{*}$ & 0,062 & $0,275^{* *}$ & $0,112^{* *}$ & $0,239^{* *}$ & 1 & \\
\hline 14. MMORPG & $0,137^{* *}$ & $-0,097^{*}$ & $0,308^{* *}$ & 0,069 & $0,252^{* *}$ & $0,501^{* *}$ & 1 \\
\hline
\end{tabular}

Nota. ${ }^{*} p<0,05 ;{ }^{* *} p<0,01$

Grupos foram formados por meio dos desvios padrões das horas de jogo semanais (HJs). Os não jogadores foram classificados como Grupo 1 e os jogadores foram divididos em grupos com distância de 0,61 DPs entre cada. Esse número foi alcançado ao dividir o intervalo total dos desvios padrões em nove seções. O número de grupos foi escolhido de modo a evitar que jogadores que apresentassem diferenças de mais de 10 horas de jogo por semana fossem categorizados nos mesmos grupos.
A configuração final dos grupos foi a seguinte: Grupo 1 $(N=160, \mathrm{HJs}=0)$, Grupo $2(N=255, \mathrm{HJ}$ entre 1 e 9$)$, Grupo 3 ( $N=159$, HJs entre 10 e 17), Grupo $4(N=94$, HJs entre 18 e 25), Grupo 5 ( $N=45$, HJs entre 26 e 32), Grupo $6(N=20, \mathrm{HJs}$ entre 33 e 40), Grupo 7 ( $N=6$, HJs entre 41 e 48), Grupo 8 ( $N=9$, HJs entre 49 e 56), Grupo $9(N=4, \mathrm{HJ}$ s entre 57 e 63), Grupo $10(N=7, \mathrm{HJ}$ s entre 64 e 74). 
Tabela 2

Correlações entre Preferências por Gênero de Jogo e Fatores de Personalidade

\begin{tabular}{|c|c|c|c|c|c|}
\hline & Socialização & Extroversão & Realização & Neuroticismo & Abertura à Experiência \\
\hline Ação & & & $0,103^{*}$ & & \\
\hline Aventura & & & & & $0,084^{*}$ \\
\hline \multicolumn{6}{|l|}{ Corrida } \\
\hline \multicolumn{6}{|l|}{ Luta } \\
\hline Puzzle & $0,093^{*}$ & & & & $0,089^{*}$ \\
\hline RPG & $-0,084^{*}$ & & $-0,108^{* *}$ & & \\
\hline Tiro & & $-0,119^{* *}$ & & & \\
\hline Simulação & & & & $0,092^{*}$ & \\
\hline Esporte & & & & $-0,182^{* *}$ & $-0,094^{*}$ \\
\hline Estratégia & & & & $-0,098^{*}$ & $0,084^{*}$ \\
\hline Música e Ritmo & $0,107^{* *}$ & $0,098^{*}$ & & & $0,095^{*}$ \\
\hline Sandbox & $-0,083^{*}$ & $-0,155^{* *}$ & $-0,098^{*}$ & & \\
\hline MOBA & & & & $-0,094^{*}$ & \\
\hline MMORPG & & & & & \\
\hline
\end{tabular}

Análises de variância foram realizadas para explorar as diferenças nos escores de bem-estar subjetivo e fatores de personalidade entre os 10 grupos. Após a ANOVA, foram realizados testes de Bonferroni para comparações múltiplas entre cada grupo. Diferenças significativas foram encontradas entre os escores de Satisfação de Vida, Afetos Positivos, Extroversão e Realização como indicados na Tabela 3.

As relações entre as horas de jogo semanais e o bem-estar subjetivo foram avaliadas também por correlações de Pearson. Entretanto, a não linearidade dos dados foi evidenciada por correlações baixas ou inexistentes. Apenas Satisfação de Vida $(r=-0,150$, $p<0,01)$, Afetos Positivos $(r=-0,087, p<0,05)$ e o fator de personalidade Realização $(r=-0,132, p<0,01)$ apresentaram correlações com as Horas de Jogo Semanais, porém, todas foram de baixo impacto, situando-se abaixo de 0,2 e representando menos de $4 \%$ de covariância.

Tabela 3

Comparação entre Grupos de Variáveis de Bem-Estar e Personalidade via Teste de Bonferroni

\begin{tabular}{|c|c|c|c|c|c|c|c|}
\hline Variável & $\begin{array}{c}\text { Sig. } \\
\text { ANOVA }\end{array}$ & Grupo (I) & Média (I) & Grupo (J) & Média (J) & $\begin{array}{l}\text { Diferença } \\
\text { média (I-J) }\end{array}$ & $\begin{array}{c}\text { Sig. } \\
\text { Bonferroni }\end{array}$ \\
\hline \multirow[t]{3}{*}{ Satisfação de Vida ${ }^{* * *}$} & ,000 & & & & & & \\
\hline & & 1 & 24,15 & 3 & 21,16 & $2,98^{* *}$ & ,003 \\
\hline & & 1 & 24,15 & 5 & 19,96 & $4,19^{* *}$ & ,009 \\
\hline \multirow[t]{3}{*}{ Afetos Positivos ${ }^{* * *}$} & ,000 & & & & & & \\
\hline & & 1 & 36,53 & 3 & 33,66 & $2,86^{*}$ & ,023 \\
\hline & & 1 & 36,53 & 4 & 33,01 & $3,51^{*}$ & ,011 \\
\hline \multirow[t]{5}{*}{ Extroversão ** } & ,002 & & & & & & \\
\hline & & 1 & 18,70 & 2 & 16,68 & $2,01^{*}$ & ,036 \\
\hline & & 1 & 18,70 & 3 & 16,09 & $2,60^{* *}$ & ,004 \\
\hline & & 1 & 18,70 & 4 & 15,84 & $2,85^{* *}$ & ,010 \\
\hline & & 1 & 18,70 & 5 & 14,80 & $3,90^{* *}$ & ,005 \\
\hline \multirow[t]{6}{*}{ Realização *** } & ,000 & & & & & & \\
\hline & & 1 & 22,12 & 2 & 20,19 & $1,93^{* * *}$ & ,001 \\
\hline & & 1 & 22,12 & 3 & 19,33 & $2,79^{* * *}$ & ,000 \\
\hline & & 1 & 22,12 & 4 & 18,94 & $3,18^{* * *}$ & ,000 \\
\hline & & 1 & 22,12 & 5 & 19,12 & $3,00^{* *}$ & ,005 \\
\hline & & 1 & 22,12 & 6 & 17,9 & $4,21^{* *}$ & ,005 \\
\hline
\end{tabular}

Nota. ${ }^{*} p<0,05 ;{ }^{* *} p<0,01 ;{ }^{* * *} p<0,001$ 


\section{Discussão}

A frequência de $76,5 \%$ participantes do gênero masculino no grupo de jogadores se manteve coerente com o apontado por outros estudos, apesar de pesquisas de público indicarem que mulheres são 58,9\% dos usuários de videogames no Brasil (Sioux Group, 2018). Predominância de participantes homens é relatada em grande parte de pesquisas envolvendo jogadores de jogos eletrônicos, com valores atingindo entre $50,5 \%$ e $98 \%$ de participantes do sexo masculino (Kaye, Carlisle, \& Griffiths, 2017; Lobel et al., 2017; Allen \& Anderson, 2018). Uma hipótese para esse fenômeno é a toxicidade da comunidade de jogadores quanto às mulheres (Brehm, 2013; Melzer, 2018). Jogar videogames ainda é considerado uma atividade masculina, sendo comuns ataques e assédios às jogadoras quando se manifestam durante partidas on-line, em fóruns e páginas dedicadas à discussões sobre jogos (Brehm, 2013). Consequentemente, esses ambientes são frequentemente escolhidos como meios de divulgação para pesquisas sobre jogadores.

Quanto às plataformas utilizadas, os valores descritos vão ligeiramente de encontro às pesquisas de mercado sobre o comportamento de jogadores. De acordo com um estudo em larga escala realizado pelo Sioux Group (2018), o equipamento mais utilizado por brasileiros para jogar são os smartphones. Esses são utilizados por $84,3 \%$ da população que se dedica a jogos eletrônicos, seguidos pelos consoles $(46,4 \%)$ e computadores $(44,6 \%)$. Na amostra deste estudo, os computadores apresentaram o maior número de usuários (71\%) seguidos pelos smartphones $(51 \%)$ e consoles da empresa Sony que foram registrados como plataforma de $46 \%$ dos jogadores. Entretanto, apenas 5,3\% dos participantes utilizam a plataforma mobile como único equipamento para jogar. É possível que essa divergência tenha se dado devido à estratégia de divulgação da pesquisa. Participantes foram recrutados por meio de comunidades e fóruns dedicados a jogos eletrônicos, porém, a maior parte dos grupos acessados era composta jogadores que utilizavam consoles e computadores como sua principal plataforma para jogar.

Quanto aos gêneros de jogo, também foram encontradas relações entre os resultados desse estudo e o que é apontado pela literatura. RPG foi o gênero considerado como favorito pelo maior número de participantes (24,9\%), seguido por Aventura (15,7\%), MMORPGs $(13,9 \%)$ e Jogos de Tiro (12\%). Na amostra de Braun et al. (2016), 46\% dos jogadores selecionaram o gênero Ação como seu favorito. Jogos de Tiro foram classificados como um subgênero de Ação, sendo os mais populares entre os subgêneros daquela categoria (61\%). RPGs foram escolhidos por $29 \%$ dos participantes e suas subcategorias preferidas pelos participantes foram os RPGs puros (41\%) e os MMORPGs
(31\%). Observa-se um padrão entre as preferências por tipos de jogos entre jogadores. Jogos de Tiro, de Ação, RPGs e MMORPGs se mantêm nas primeiras posições. $\mathrm{Na}$ amostra apresentada, a preferência por RPGs possui relação significativa com jogos de Aventura $(r=0,351$, $p<0,01)$ e MMORPGs $(r=0,501, p<0,01)$ o que pode explicar a prevalência desses três tipos de jogos entre os favoritos. A amostra possivelmente foi composta por indivíduos com mais inclinação a preferir os gêneros citados.

Quanto às hipóteses, a primeira investigada foi: A personalidade dos jogadores possui relação com as preferências por gêneros de jogos eletrônicos. A hipótese nula foi rejeitada pelos resultados das análises. Os fatores de personalidade apresentaram algumas relações com as preferências por tipos de jogos. Indivíduos mais abertos a novas experiências se mostraram inclinados a jogar jogos de Aventura, Puzzle, Estratégia e de Música e Ritmo, e a evitar jogos de Esporte. Em outros estudos, maior Abertura à Experiência apresentou relações com a preferência por RPGs e probabilidade de experienciar maiores níveis de bem-estar decorrentes do uso de jogos eletrônicos (Johnson \& Gardner, 2010; Braun et al., 2016). Neuroticismo apresentou correlações positivas com jogos de Simulação e negativas com jogos de Esporte, Estratégia e MOBAs. A relação positiva com jogos de simulação apresenta-se também em outro estudo (Braun et al., 2016). Extroversão relacionou-se positivamente com jogos de Música e Ritmo e negativamente com jogos de Tiro e Sandbox. Esses dados divergem do encontrado na literatura que aponta que jogadores com maior extroversão possuem preferência por Jogos de Tiro e de Ação, já que eles os permitem entrar em contato com outros em partidas multiplayer (Braun et al., 2016; Jimenez et al., 2019). Porém, apesar de relações significativas terem sido encontradas entre determinados tipos de jogos e os fatores de personalidade, deve-se considerar que todas as relações (em módulo) situaram-se em um valor abaixo de 0,2 , o que as configura como muito fracas (Evans, 1996).

Diferenças entre os fatores de personalidade Realização e Extroversão foram observadas quando comparados os grupos estipulados por meio das Horas de Jogo Semanais (HJs). O grupo de não jogadores (1) apresentou maiores médias em ambos os fatores quando comparados com os grupos de jogadores. Os escores de Extroversão foram maiores no primeiro quando em comparação com os grupos 2 ao 5 (de 1 à $32 \mathrm{HJs}$ ) e os de Realização, também maiores entre os não jogadores, quando comparados com os grupos 2 ao 6 (de 1 à $40 \mathrm{HJs}$ ) e o grupo 10 (entre 64 e $74 \mathrm{HJs}$ ). Esses dados corroboram o apontado pela literatura, de que indivíduos com menores níveis de Extroversão demonstram preferência por atividades de pouca interação com outras pessoas, como os jogos eletrônicos (McCrae \& Costa, 2008; Lehenbauer-Baum et al., 2015). Jogadores 
apresentam menores níveis de extroversão em diversos estudos, quando comparados com indivíduos que não jogam (Shen \& Williams, 2011; Lehenbauer-Baum et al., 2015; Braun et al., 2016). Realização foi outro fator que exibiu maiores escores entre os não jogadores. Realização é o fator que faz referência a questões de controle, organização e persistência para o alcance de objetivos. Indivíduos que passam maior parte do dia engajados em jogos eletrônicos têm demonstrado menores níveis de Realização e maior probabilidade de apresentarem uso problemático de videogames (Braun et al., 2016). Entretanto, é delicado generalizar devido à grande variedade e o número de contextos envolvendo jogos eletrônicos. Profissionais de E-Sports (jogos competitivos para os quais são organizados campeonatos) apresentam altos escores de Realização quando comparados com outros jogadores e não jogadores (Martončik, 2015). Na amostra analisada por esta pesquisa não houve diferenças significativas entre os grupos de jogadores.

A segunda hipótese estimava que os escores de afetos positivos e satisfação de vida seriam maiores em jogadores que se dedicam moderadamente aos jogos eletrônicos, em comparação com os que não jogam, jogam pouco ou intensamente. Os dados apresentados indicam que não se pode rejeitar a hipótese nula nessa amostra. Apesar de diferenças serem apontadas entre o grupo de não jogadores e alguns grupos de jogadores, os escores nas escalas de bem-estar subjetivo se mantiveram constantes entre os participantes que dedicaram mais de uma hora por semana aos jogos eletrônicos. Quanto às variáveis, foram relatados maiores níveis de Afetos Positivos e Satisfação de vida no Grupo 1 quando em comparação com os grupos 3, 5 e 10 (Satisfação de Vida) e com os grupos 3 e 4 (Afetos Positivos). Os Afetos Negativos se mantiveram estáveis entre todos os grupos. Não houve diferenças, nas variáveis de bem-estar, entre os não jogadores e o Grupo 2 (de uma a nove $\mathrm{HJs}$ ), podendo evidenciar que o uso de jogos eletrônicos por até nove horas por semana não indica nenhuma alteração nos níveis de bem-estar no que se refere às comparações entre jogadores e não-jogadores. Porém, não foram encontradas diferenças entre os grupos.

A terceira hipótese considerou que a frequência de jogo em horas semanais se relaciona com as variáveis de bem-estar subjetivo e com fatores de personalidade. Não se pode rejeitar a hipótese alternativa devido à existência dessas relações. Entretanto, corrobora o apontado por outros estudos, nos quais as relações das horas de jogo com as variáveis de bem-estar e personalidade, quando existentes, mostraram-se fracas (Jackson et al., 2010; Allen \& Anderson, 2018; Twenge, Martin, \& Campbell, 2018). Esses resultados podem ser indicativos de que há outros fatores para além da frequência de jogo que possuem maior influência no bem-estar. Alguns autores apontam que motivações sociais, de fuga da realidade ou de satisfação das necessidades psicológicas básicas apresentam impacto significativo nas variáveis de bem-estar (Vella et al., 2015; Comello et al., 2016; Ladeira et al, 2016). É recomendado que esses fatores sejam considerados ao avaliar o bem-estar de jogadores.

Esse estudo investigou o uso de jogos eletrônicos e as relações entre bem-estar, personalidade, frequência de uso de jogos eletrônicos e gêneros de jogos. Entretanto, algumas limitações foram encontradas e devem ser consideradas para interpretação e generalização dos dados. A amostra, apesar de ser composta por indivíduos de todas as regiões do Brasil, teve aproximadamente $70 \%$ dos participantes habitando a região Sul do país, sendo pouco representativa da população brasileira. Ademais, deve-se considerar a possibilidade de diferenças entre gêneros influenciarem os resultados das análises de comparação entre grupos.

Outra limitação foi a escolha do número de grupos dos jogadores, prejudicando as análises dos que apresentaram maiores horas de jogos por semana. Ademais, cada jogo e gêneros de jogos possuem características, demandam tempo de dedicação e habilidades distintas e possuem públicos-alvo diferentes. A generalização de informações sobre pessoas que jogam jogos eletrônicos deve ser um procedimento cauteloso, visto que existem tipos de jogadores diferentes com motivações e interesses específicos (Braun et al., 2016).

Para estudos futuros, recomenda-se melhorar a representatividade na amostra, buscando maior equilíbrio quanto ao gênero e distribuição geográfica de participantes; investigar as relações entre personalidade e horas de jogo apontadas nesse estudo em indivíduos que jogam videogames como profissão, comparando-os com os que jogam como meio de lazer. Adicionalmente, deve-se focar nas motivações que levam as pessoas a dedicarem tempo aos jogos eletrônicos. O estudo das motivações para jogar é fundamental para que se possa compreender as relações entre jogos eletrônicos e variáveis de bem-estar (Comello et al., 2016; Allen \& Anderson, 2018).

Jogos eletrônicos são um dos meios de entretenimento mais populares e rentáveis do mundo. No Brasil, 75,7 milhões de pessoas gastaram em torno de 1,3 bilhões de dólares com a indústria de videogames no ano de 2018 (Newzoo, 2018). Culpabilizar os jogos como causadores de malefícios para a saúde mental de seus usuários e generalizar todos os jogadores como um grupo único com características exclusivas são falácias comuns cometidas por pesquisadores e divulgadas na mídia (Bean, Nielsen, van Rooij, \& Ferguson, 2017). São poucos os indícios de que o número de horas de uso de jogos eletrônicos, isoladamente, acarrete em algum impacto significativo no bem-estar subjetivo de jogadores. Alguns autores apontam maior influência dos motivos para jogar na variável bem-estar (Vella et al., 2015; Comello et al., 2016; Allen \& Anderson, 2018). 
Pesquisas sobre as relações entre a utilização de jogos eletrônicos e o bem-estar de seus usuários vêm se tornando mais frequentes e necessárias para uma maior compreensão desse fenômeno mundial. Essa discussão é necessária para que se possa evitar a patologização dos jogos de forma generalista, visto que videogames já se mostraram efetivos em uma série de intervenções para o ensino e saúde, sendo suas potencialidades amplamente investigadas (Boyle et al., 2016). Ao focar apenas na frequência de uso como a variável que se relaciona com a saúde mental, perde-se a complexidade dos processos que levam um indivíduo a engajar-se de forma saudável ou problemática em qualquer atividade, incluindo jogar videogames (Bean et al., 2017; Allen \& Anderson, 2018).

\section{Referências}

Allen, J. J., \& Anderson, C. A. (2018). Satisfaction and frustration of basic psychological needs in the real world and in video games predict internet gaming disorder scores and well-being. Computers in Human Behavior, 84, 220-229. doi: 10.1016/j.chb.2018.02.034

Anglim, J., \& Grant, S. (2016). Predicting psychological and subjective well-being from personality: Incremental prediction from 30 facets over the Big 5. Journal of Happiness studies, 17(1), 59-80. doi: 10.1007/s10902-014-9583-7

Bean, A. M., Nielsen, R. K. L., van Rooij, A. J., \& Ferguson, C. J. (2017). Video game addiction: The push to pathologize video games. Professional Psychology: Research and Practice, 48(5), 378-389. doi: 10.1037/pro0000150

Błachnio, A., Przepiorka, A., \& Pantic, I. (2016). Association between Facebook addiction, selfesteem and life satisfaction: A cross-sectional study. Computers in Human Behavior, 55, 701-705. doi: 10.1016/j.chb.2015.10.026

Boyle, E. A., Hainey, T., Connolly, T. M., Gray, G., Earp, J., Ott, M., ... \& Pereira, J. (2016). An update to the systematic literature review of empirical evidence of the impacts and outcomes of computer games and serious games. Computers \& Education, 94, 178-192. doi: 10.1016/j.compedu.2015.11.003

Braun, B., Stopfer, J. M., Müller, K. W., Beutel, M. E., \& Egloff, B. (2016). Personality and video gaming: Comparing regular gamers, non-gamers, and gaming addicts and differentiating between game genres. Computers in Human Behavior, 55, 406-412. doi: 10.1016/j. chb.2015.09.041

Brehm, A. L. (2013). Navigating the feminine in massively multiplayer online games: gender in World of Warcraft. Frontiers in psychology, 4, 1-12. doi: 10.3389/fpsyg.2013.00903

Carras, M. C., Van Rooij, A. J., de Mheen, D., Musci, R., Xue, Q.-L., \& Mendelson, T. (2017). Video gaming in a hyperconnected world: A cross-sectional study of heavy gaming, problematic gaming symptoms, and online socializing in adolescents. Computers in Human Behavior, 68, 472-479. doi: 10.1016/j.chb.2016.11.060

Comello, M. L. G., Francis, D. B., Marshall, L. H., \& Puglia, D. R. (2016). Cancer survivors who play recreational computer games: Motivations for playing and associations with beneficial psychological outcomes. Games for Health Journal, 5(4), 286-292. doi: 10.1089/ g4h.2016.0003

Delle Fave, A., Massimini, F., \& Bassi, M. (2011). Hedonism and eudaimonism in positive psychology. Em Psychological selection and optimal experience across cultures (pp. 3-18). Springer, Dordrecht. doi: 10.1007/978-90-481-9876-4_1

Diener, E. (1984). Subjective well-being. Psychological bulletin, 95(3), 542.

Diener, E. D., Emmons, R. A., Larsen, R. J., \& Griffin, S. (1985). The satisfaction with life scale. Journal of personality assessment, $49(1), 71-75$. doi: 10.1207/s15327752jpa4901_13

Diener, E., Heintzelman, S. J., Kushlev, K., Tay, L., Wirtz, D., Lutes, L. D., \& Oishi, S. (2017). Findings all psychologists should know from the new science on subjective well-being. Canadian Psychology/psychologie canadienne, 58(2), 87. doi: 10.1037/cap0000063

Diener, E., Tay, L., \& Oishi, S. (2013). Rising income and the subjective well-being of nations. Journal of personality and social psychology, 104(2), 267. doi: $10.1037 / \mathrm{a} 0030487$

Evans, J. D. (1996). Straightforward statistics for the behavioral sciences. Pacific Grove, CA: Brooks/Cole Publishing.

Granic, I., Lobel, A., \& Engels, R. C. (2013). The benefits of playing video games. American psychologist, 69(1), 66. doi: 10.1037/a0034857

Hutz, C. S., Zanon, C., \& Bardagi, M. P. (2014). Satisfação de Vida. Em Hutz, C. S. (Eds.), Avaliação em Psicologia Positiva (pp. 43-47). São Paulo, Brasil: Artmed.

Jackson, L., Witt, E. A., Von Eye, A., Fitzgerald, H. E., \& Zhao, Y. (2010). Children's information technology (IT) use and their physical, cognitive, social and psychological well-being. In 2010 Fourth International Conference on Digital Society (pp. 198-203). IEEE. Michigan State University: Michigan, United States. doi: 10.1109/ICDS.2010.69

Jimenez, N., San-Martin, S., Camarero, C., San, R., Cabezudo, J., Jimenez, N., \& San-Martin, S. (2019). What kind of video gamer are you? Journal of Consumer Marketing, 36(1), 218-227 doi: 10.1108/JCM-06-2017-2249

Johnson, D., \& Gardner, J. (2010) Personality, motivation and video games. In Proceedings of the 22nd Conference of the Computer-Human Interaction Special Interest Group of Australia on Computer-Human Interaction (pp. 276-279). Brisbane, Australia: Association for Computing Machinery, Inc

Kaye, L. K., Carlisle, C. R., \& Griffiths, L. R. W. (2017). A contextual account of the psychosocial impacts of social identity in a sample of digital gamers. Psychology of Popular Media Culture, 8(3), 259. doi: 10.1037/ppm0000173

Ladeira, W. J., de Santini, F. O., Sampaio, C. H., \& Araujo, C. F. (2016). Experiential value and domain-specific innovativeness during freemium game usage: effects on child well-being. Young Consumers, 17(1), 64-77. doi: 10.1108/YC-07-2015-00538

Lee, J. H., Karlova, N., Clarke, R. I., Thornton, K., \& Perti, A. (2014). Facet Analysis of Video Game Genres. In iConference 2014 Proceedings (p. 125-139). doi:10.9776/14057

Lehenbauer-Baum, M., Klaps, A., Kovacovsky, Z., Witzmann, K., Zahlbruckner, R., \& Stelina, B. U. (2015). Addiction and engagement: An explorative study toward classification criteria for internet gaming disorder. Cyberpsychology, Behavior, and Social Networking, 18(6), 343-349. doi: 10.1089/cyber.2015.0063 
Lobel, A., Engels, R. C. M. E., Stone, L. L., Burk, W. J., \& Granic, I. (2017). Video gaming and children's psychosocial wellbeing: A longitudinal study. Journal of Youth and Adolescence, 46(4), 884-897. doi: 10.1007/s10964-017-0646-z

Martončik, M. (2015). e-Sports: Playing just for fun or playing to satisfy life goals? Computers in Human Behavior, 48, 208-211. doi:10.1016/j. chb.2015.01.056

McCrae, R. R., \& Costa, P. T., Jr. (2008). Empirical and theoretical status of the FiveFactor Model of personality traits. In G. J. Boyle, G. Matthews, \& D. H. Saklofske (Eds.). The SAGE handbook of personality theory and assessment (pp. 273-294). Los Angeles, CA: SAGE.

Melzer, A. (2018). Of princesses, paladins, and players: Gender stereotypes in video games. Em Evolutionary Psychology and Digital Games (pp. 205-220). Routledge.

Natividade, J. C., \& Hutz, C. S. (2015). Escala reduzida de descritores dos cinco grandes fatores de personalidade: prós e contras. Psico, 46(1), 79-89.

Newzoo (2018). Brazil Games Market 2018. Recuperado de https://newzoo.com/insights/infographics/brazil-games-market-2018/

Sioux Group (2018). Pesquisa Games Brasil 18. Recuperado de https://bit.ly/2Ej3fQm

Scharkow, M., Festl, R., Vogelgesang, J., \& Quandt, T. (2015). Beyond the "core-gamer": Genre preferences and gratifications in computer games. Computers in Human Behavior, 44, 293-298.

Shen, C., \& Williams, D. (2011). Unpacking time online: Connecting internet and massively multiplayer online game use with psychosocial well-being. Communication Research, 38(1), 123-149. doi: 10.1177/0093650210377196

Soto, C. J. (2015). Is happiness good for your personality? Concurrent and prospective relations of the big five with subjective well-being. Journal of personality, 83(1), 45-55. doi: 10.1111/jopy.12081

Twenge, J. M., Martin, G. N., \& Campbell, W. K. (2018). Decreases in Psychological Well-Being Among American Adolescents After 2012 and Links to Screen Time During the Rise of Smartphone Technology. Emotion, 18(6), 765-780. doi: 10.1037/emo0000403

Vella, K., Johnson, D., \& Hides, L. (2015). Playing alone, playing with others: Differences in player experience and indicators of wellbeing. In Proceedings of the 2015 annual symposium on computer-human interaction in play (pp. 3-12). Brisbane, Australia: Association for Computing Machinery, Inc. doi: 10.1145/2793107.2793118

Zammitto, V. L. (2010). Gamers' personality and their gaming preferences (Tese de doutorado). Communication, Art \& Technology: School of Interactive Arts and Technology.

Zanon, C., Bastianello, M. R., Pacico, J. C., \& Hutz, C. S. (2013). Desenvolvimento e validação de uma escala de afetos positivos e negativos. Psico-USF, 18(2), 193-201.

\section{Sobre os autores}

Fábio Spricigo Coser é psicólogo (UFRGS) e mestre em Psicologia pela Universidade Federal do Rio Grande do Sul (UFRGS).

Claudia Hofheinz Giacomoni é psicóloga (PUC-RS), doutora em Psicologia pela Universidade Federal do Rio Grande do Sul (UFRGS) e, atualmente, é professora no Programa de Pós-Graduação Stricto Sensu em Psicologia da UFRGS. 